\title{
La pandemia de coronavirus SARS-CoV-2 (COVID-19): situación actual e implicaciones para México
}

\author{
The SARS-CoV-2 (COVID-19) coronavirus pandemic: \\ current situation and implications for Mexico \\ Xavier Escudero, * Jeannette Guarner, ${ }^{\ddagger}$ Arturo Galindo-Fraga, ${ }^{\S}$ \\ Mara Escudero-Salamanca," Marco A Alcocer-Gamba," Carlos Del-Río**
}

\footnotetext{
Palabras clave: Coronavirus,

COVID-19,

SARS-CoV-2,
} pandemia, México.

Keywords: Coronavirus, COVID-19, SARS-CoV-2, pandemic, Mexico.

* Servicio de Cardiología. Hospital Médica Sur. American College of Cardiology Capítulo México. Ciudad de México, México.

‡ Departamento de Patología, Escuela de Medicina, Universidad de Emory.

Atlanta, EUA.

§ Subdirección de Epidemiología Hospitalaria y Control de Calidad de la Atención Médica, Instituto Nacional de Ciencias Médicas y Nutrición «Salvador Zubirán». Ciudad de México, México.

\section{RESUMEN}

La pandemia del Coronavirus (COVID-19) es una de las más devastadoras de este siglo. Originada en China en diciembre de 2019 y causada por el virus SARS-CoV-2, en menos de un mes ya había sido catalogada como «Emergencia de Salud Pública de Alcance Internacional». A la fecha hay cerca de tres millones de personas con infección confirmada y ha provocado más de 250,000 fallecimientos en el mundo. Inicialmente afecta las vías respiratorias con neumonías atípicas y en casos graves provoca inflamación sistémica con liberación de citoquinas que pueden provocar un rápido deterioro, insuficiencia circulatoria, respiratoria y alteraciones de coagulación con una letalidad cercana a 7\%. En México, el primer caso se detectó en febrero de 2020, y a la fecha de esta publicación se cuenta con 29,616 casos confirmados y 2,961 fallecimientos en toda la extensión del país. La baja tasa de muestreo diagnóstico en nuestro país claramente subestima la incidencia e impacto de esta enfermedad. Los grupos más afectados son aquéllos con factores de riesgo como lo son la edad mayor a 60 años, hipertensión, diabetes o historia de enfermedad cardiovascular. De los casos confirmados, $15 \%$ son trabajadores del sector salud. No existe hasta ahora un tratamiento específico o vacuna, de tal manera que es importante contar con las medidas de higiene, aislamiento social y protección personal. Las consecuencias en salud, sociales y económicas podrían ser de gran impacto en los tiempos por venir.

\section{ABSTRACT}

The Coronavirus pandemic (COVID-19) is one of the most devastating in this century. It originated in China in December 2019 caused by the SARS-CoV-2 virus, and in less than a month it had been classified as an «International Public Health Emergency». To date there are nearly3 million people infected and more than 250,000 deaths caused by the disease worldwide. Initially it affects the respiratory tract with atypical pneumonia and in severe cases it produces systemic inflammation with cytokine storm that can cause rapid deterioration with circulatory and respiratory failure, coagulopathy and a lethality rate of approximately $7 \%$. In Mexico, the first case was detected in February 2020, and to date there are 26,616 confirmed cases and 2,961 deaths throughout the country. The low number of diagnostic tests conducted in our country clearly underestimates the real incidence and impact of the disease. The most affected groups are those with risk factors such as age over 60, presence of hypertension, diabetes or cardiovascular disease. Of the confirmed cases, $15 \%$ are healthcare workers. There is no specific treatment or vaccine yet, so it is important to have hygiene, social isolation and personal protection measures. Health, social and economic consequences could have great impact in the near future.

Cuando queda establecida la permanencia (epidemia) de una enfermedad concreta, está claro que la forma de vida es su causa y que el aire que respiramos es el culpable.

Hipócrates de Cos (460-370 a. C.)

De Natura Hominis 
\| Departamento de Cardiología Nuclear, Instituto Nacional de Cardiología «Ignacio Chávez», Facultad Mexicana de Medicina de la Universidad La Salle. Ciudad de México, México.

ฯ Jefatura de Cardiología

Intervencionista, Instituto del Corazón de Querétaro, Facultad de Medicina de la Universidad Autónoma de Querétaro. Ciudad de Querétaro, Querétaro, México.

** Departamento de Medicina, División de Enfermedades Infecciosas. Escuela de Medicina, Universidad de Emory. Atlanta, EUA.

Recibido: 24/04/2020 Aceptado: 29/04/2020
D esde su origen, el hombre ha sido víctima de graves infecciones y pandemias que a través de los tiempos han sido una de las principales causas de muerte. Las más devastadoras son siempre aquéllas que surgen en «brotes» provocados por nuevos microorganismos que afectan a un amplio segmento de la población. Desde la plaga de Justiniano en el siglo VI d. C., o la «peste negra» en el siglo XIV, hasta la pandemia del virus de la inmunodeficiencia humana $(\mathrm{VIH})$ en el siglo XX, las epidemias han provocado la muerte de cientos de millones de personas. La gran pandemia en tiempos recientes fue la de la «influenza española» en 1918, que infectó a 500 millones de habitantes con 50 millones de muertes. ${ }^{1}$ Aún vivimos el impacto del VIH/SIDA que ha infectado a más de 72 millones de seres humanos y causado 32 millones de muertes desde el inicio de la pandemia. ${ }^{2}$ En 2003, en China, apareció un nuevo coronavirus y se inició la epidemia del SARS (síndrome respiratorio agudo grave) provocada por el coronavirus SARS-CoV que afectó alrededor de 8,000 personas y causó más de 700 fallecimientos. En 2009, la llamada «gripe porcina» causada por un nuevo virus de la influenza A (H1N1) causó la muerte de cerca de 500,000 personas, y en 2012 la epidemia de MERS (síndrome respiratorio del Oriente Medio) fue producto de un nuevo coronavirus (MERS-CoV) con 2,500 casos reportados. Con los brotes de ébola en África, en particular el que ocurrió en 2013-2014 en Sierra Leona, Nueva Guinea y Liberia, las fiebres hemorrágicas fueron temidas en el resto del mundo, ya que hubo pacientes con la enfermedad en Europa y Estados Unidos. ${ }^{3}$ La vigilancia epidemiológica y el haber podido contener los casos logró que no se expandiera la enfermedad. En 2015-2016, el virus Zika tuvo consecuencias devastadoras en América del Sur debido a sus efectos en el sistema nervioso central, con malformaciones congénitas importantes y fue considerada por la Organización Mundial de la Salud (OMS) «emergencia de salud pública con alcance internacional», y en años subsecuentes disminuyó su transmisión. ${ }^{4,5}$

Una nueva pandemia de origen viral era temida y esperada a nivel mundial desde hace varios años. Y así ocurrió: el 31 de diciembre de 2019, el Ministerio de Salud de China informó a la OMS sobre 41 pacientes con «neumonía atípica grave», la mayoría de ellos relacionados con el mercado de comida de mariscos y animales exóticos en la ciudad de Wuhan, en la provincia de Hubei en China. ${ }^{6}$ Este fue el inicio de lo que ahora es una de las pandemias más devastadoras de los últimos tiempos.

\section{EL SARS-COV-2 Y LA PANDEMIA COVID-19}

\section{El agente causal}

El virus es conocido como SARS-CoV-2, un nuevo betacoronavirus de la familia de los Coronaviridae, Ilamado así por su cápsula lipoproteica de forma esférica rodeada de múltiples espículas (glicoproteínas-S) que le dan aspecto de corona. El material genético en su interior es una sola cadena de ácido ribonucleico (ARN) de sentido positivo. La proximidad genética con dos coronavirus presentes en los murciélagos hace altamente probable que ese sea su origen, con la posible participación de uno o varios hospederos intermediarios..$^{7,8} \mathrm{El}$ virus ingresa usualmente por vía respiratoria (aun cuando lo puede hacer a través de las mucosas como la conjuntiva) y se fija mediante las espículas a su receptor: la proteína de membrana enzima convertidora de angiotensina tipo 2 (ECA-2) de las células del epitelio y alveolares tipo II. Una vez interiorizado, el ARN es liberado para su transcripción y replicación.

\section{La enfermedad}

La OMS, Ilamó a esta enfermedad «COVID-19» (COronaVIrus Disease 2019) el $11 \mathrm{de}$ febrero de 2020 por el tipo de virus y el año de aparición. Esta infección es principalmente transmitida de humano a humano por contacto mediante gotas de Flügge (secreciones respiratorias $>5 \mu$ ) y en ocasiones también por aerosoles. A pesar deser unvirus envuelto, lo que lo hace relativamente inestable, puede permanecer en superficies de plástico o metal de 24 hasta 72 horas. Su índice de contagio es alto $\left(\mathrm{R}_{0}=\right.$ número de reproducibilidad hasta 5), lo que hace que una persona infectada pueda contagiar de dos a cinco personas, con lo cual se explica su crecimiento exponencial. ${ }^{9}$ El periodo de incubación es de uno a 14 días con una media de cinco a seis días. 
La información sobre características clínicas generales reportadas varían de acuerdo con las series y se basan fundamentalmente en los casos de pacientes hospitalizados. ${ }^{6,10-14}$ En la serie de China, la edad promedio fue de 47 años, en comparación con los datos de España donde fue de 58 o en Italia con promedio de 64 años. ${ }^{12,13}$ Hay una ligera predominancia del género masculino (51-59\%) en las diferentes series. Los signos y síntomas más frecuentes fueron: fiebre (>90\%), tos seca $(70 \%)$ y dificultad respiratoria $(37 \%)$. Hubo presentación variable de otros síntomas como fatiga/astenia (38-69\%), mialgias, cefalea, congestión conjuntival o diarrea en pocos casos. ., $^{81}$ La presencia de comorbilidades también fue variable en los diferentes reportes, con presencia de diabetes de 13 a 32\%, hipertensión arterial de 18 a 49\% y presencia de enfermedad cardiovascular de 4 hasta $42 \%$. Esta gran variación se debe al número de pacientes estudiados, las diferencias en la edad de la población afectada y el estado de gravedad en el momento de la inclusión en cada serie ${ }^{11,13,14}$ (Tabla 1).

En cuanto a los estudios de laboratorio, fue frecuente la presencia de linfocitopenia (80\%) y trombocitopenia (36\%). La elevación del dímero-D presente en $46 \%$ de los pacientes hospitalizados y la elevación de la troponina son marcadores de mal pronóstico. Los hallazgos radiográficos o tomográficos son frecuentes (84\%) y consisten en imágenes focales unilaterales o bilaterales en «vidrio deslustrado», infiltrados micronodulares en

Tabla 1: Edad, género y comorbilidades en pacientes con COVID-19 en varias series internacionales. ${ }^{11-14}$

\begin{tabular}{lcccc} 
& $\begin{array}{c}\text { China } \\
\mathrm{n}=1,099\end{array}$ & $\begin{array}{c}\text { España } \\
\mathrm{n}=18,609\end{array}$ & $\begin{array}{c}\text { Italia* } \\
\mathrm{n}=1,043\end{array}$ & $\begin{array}{c}\text { EUA* } \\
\mathrm{n}=1,069\end{array}$ \\
\hline Edad (años) & 47.0 & 58 & 64 & 54 \\
Género masculino & 58.0 & 51 & 59 & 58 \\
Diabetes & 16.0 & 13 & 17 & 32 \\
Hipertensión & 24.0 & 18 & 49 & 34 \\
Enfermedad cardiovascular & 4.0 & 8 & 30 & 23 \\
EPOC & 3.5 & 11 & 4 & 21 \\
\hline
\end{tabular}

* Pacientes de la Unidad de Terapia Intensiva.

EPOC = enfermedad pulmonar obstructiva crónica. parche, zonas de consolidación o derrame pleural. ${ }^{11}$ El diagnóstico se confirma con la prueba de RT-PCR (reacción en cadena de polimerasa por transcriptasa reversa en tiempo real) de muestra por hisopado directo de mucosa nasofaríngea, secreciones o aspirado bronquial. El pronóstico y la letalidad son muy variables. En el registro del Centro de Control y Prevención de Enfermedades de China de pacientes hospitalizados, la mayoría (81\%) fueron reportados con afección leve, $14 \%$ con afección severa y $5 \%$ con afección crítica. La mortalidad general fue de $2.3 \%$ que se incrementó a $8 \%$ en pacientes mayores de 80 años, $10 \%$ en aquéllos con enfermedad cardiovascular y $49 \%$ de mortalidad en los pacientes en estado crítico. ${ }^{14}$

\section{La pandemia mundial}

Desde los primeros casos reportados en Wuhan, China, la expansión local y finalmente mundial llegó rápidamente a niveles alarmantes. Para el 11 de enero de 2020 ya había cobrado la primera víctima mortal y un mes después, el 11 de febrero, el Centro de Control y Prevención de Enfermedades de China tenía 72,314 casos reportados. ${ }^{14}$ El 30 de enero de 2020, la OMS declaró al COVID-19 como «emergencia de salud pública de alcance internacional», y el 11 de marzo con 37,364 casos reportados fuera de China, se catalogó oficialmente como «pandemia». Por primera vez en la historia se ha dado seguimiento a esta enfermedad en «tiempo real» a través de las diferentes plataformas digitales, con estadísticas y datos detallados día a día y minuto a minuto. $\mathrm{Al}$ momento de esta publicación, en el mundo, se han reportado más de cuatro millones de casos y 300,000 muertes en 214 países, lo que aporta una letalidad global cercana a $7 \% .{ }^{15} \mathrm{El}$ comportamiento de las curvas epidemiológicas de casos positivos y de mortalidad han sido muy variables en diferentes regiones (Figura 1). ${ }^{22}$ Llama la atención el marcado crecimiento e impacto en ciertos países como Italia, España y sobre todo en Estados Unidos de América, donde a la fecha hay alrededor de 1,400,000 casos confirmados y más de 86,000 muertes. Esta gran diferencia en la velocidad de crecimiento y magnitud del impacto depende de múltiples variables 


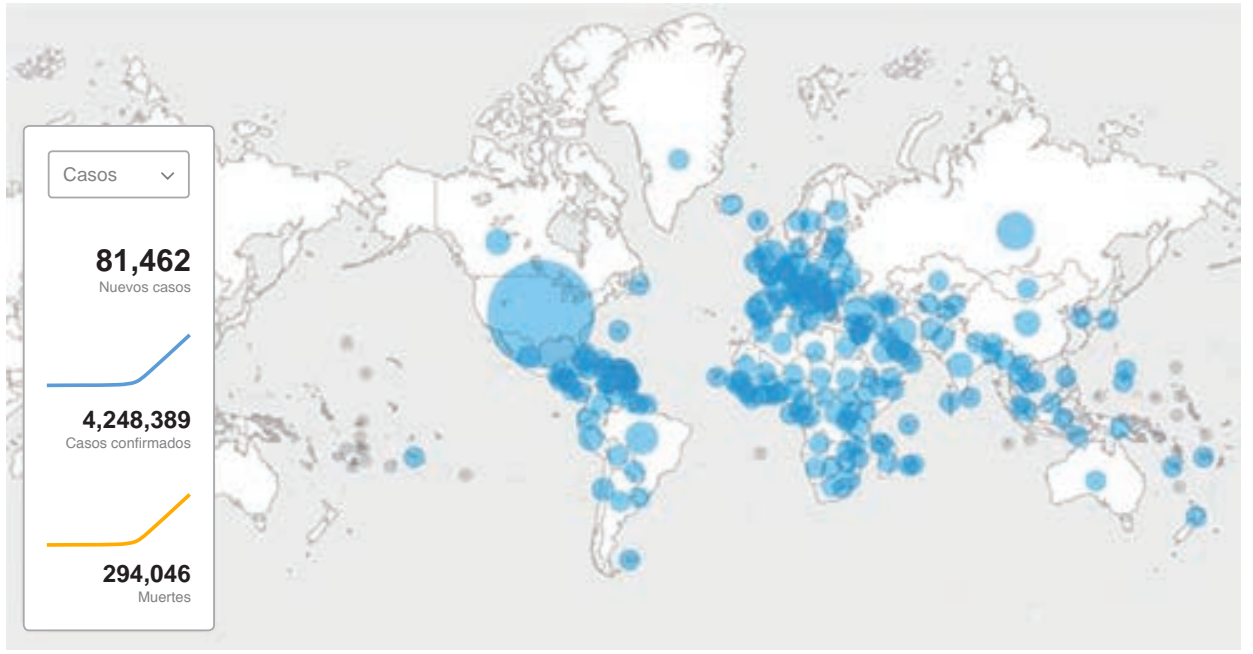

\section{Figura 1:}

Impacto mundial por la pandemia de COVID-19. Número de casos confirmados, nuevos y muertes reportadas por país (actualizado al 14 de mayo de 2020). ${ }^{15}$ como lo son la edad promedio en la población afectada, prevalencia de factores de riesgo, aspectos raciales, sociales y climáticos, pero sobre todo en los sistemas de salud de cada país y de las diversas estrategias para la aplicación de medidas de detección y prevención. Estamos sin duda ante un fenómeno epidemiológico y de salud sin precedentes para este siglo y las consecuencias sanitarias, sociales y económicas permanecerán por mucho tiempo.

\section{El uso de pruebas diagnósticas}

Durante la fase aguda de la infección el virus se puede encontrar utilizando pruebas que detectan ácidos nucleicos en secreciones de la nasofaringe. ${ }^{16}$ Generalmente se amplifican dos genes blanco (RdPR y E, en el llamado protocolo Berlín, que es el que se corre en México $)^{17}$ y se utiliza un gen humano para definir que la prueba funcionó de manera adecuada. Lo que es importante reconocer es que la presencia de ácidos nucleicos no necesariamente indica que el virus es viable. Para determinar la viabilidad es necesario hacer cultivos virales, que en realidad sólo son realizados en laboratorios de referencia especializados en virología como es el Centro de Control y Prevención de Enfermedades (CDC) en los Estados Unidos. La duración del virus en la nasofaringe no es del todo conocida en la actualidad y es posible que dependa de varios factores relacionados con el huésped. En un estudio europeo se encontró virus reproducible hasta por siete días en secreciones respiratorias, teniendo detección de ácidos nucleicos hasta por 21 días. ${ }^{18}$ En la fase de convalecencia lo que se encuentra son anticuerpos en suero anti-SARS-CoV-2. Estos anticuerpos pueden ser IgM que generalmente aparecen después de la invasión a nasofaringe o lgG que se detectan después de la IgM. Algunos han buscado también IgA anti SARS-CoV-2, ya que al ser una enfermedad inicialmente de la mucosa respiratoria, este tipo de respuesta es pronunciada. Lo cierto es que la realización de pruebas de detección es clave para conocer la magnitud real de la pandemia y ayudar a contener su expansión.

\section{Evaluación del paciente}

Aproximadamente $80 \%$ de loscasos son leves y por lo tanto no requieren de hospitalización ni de alguna intervención terapéutica. En dichos pacientes se recomienda su manejo en casa donde deben permanecer en aislamiento estricto por aproximadamente siete días después del inicio de síntomas o 72 horas luego de la resolución de la fiebre sin antipirético. Es importante recalcar que un porcentaje de pacientes que inicialmente tienen síntomas menores pueden tener un deterioro subsecuente en la primera semana, por lo que aquéllos con factores de riesgo para enfermedad severa requieren de vigilancia estrecha. En los pacientes con disnea quetienen hipoxemia y/o infiltrados pulmonares se recomienda la hospitalización, lo que ocurre 
en $20 \%$ de los pacientes positivos. La edad y la presencia de comorbilidades, afección cardiaca o alteraciones de coagulación son marcadores de alto riesgo, y de los pacientes quellegan a requerir de apoyo de ventilación mecánica el $80 \%$ de ellos fallece. ${ }^{8}$

\section{Perspectivas terapéuticas}

Hoy en día no existe ningún medicamento que se haya aprobado para el tratamiento del SARS-CoV-2. Varios fármacos y estrategias se han utilizado, pero no existe evidencia para recomendar ninguno de ellos fuera de un ensayo clínico. Entre los medicamentos que han sido utilizados está la hidroxicloroquina/ cloroquina con o sin azitromicina y el lopinavir/ ritonavir, hasta ahora sin claros beneficios y con potenciales efectos secundarios. ${ }^{19,20}$ Hay otros antivirales en investigación incluyendo el remdesivir, o inmunomoduladores como el tocilizumab. Otra modalidad terapéutica que se ha utilizado es el plasma de pacientes convalecientes. En teoría, aquellas personas que han sobrevivido a la enfermedad tienen anticuerpos anti-SARS-CoV-2 que pueden ayudar a tratar a otros enfermos. Al igual que otras modalidades terapéuticas ésta tampoco ha sido investigada en ensayos clínicos aleatorizados. Un punto importante de mencionar es sobre el uso de inhibidores de la ECA y bloqueadores del receptor de angiotensina. No existe indicación para su uso específico en COVID-19, pero si estos medicamentos están siendo utilizados para el manejo de hipertensión o insuficiencia cardiaca, éstos no deben ser suspendidos, ya que su uso no está asociado a mayor propensión o gravedad de la enfermedad y de hecho parecen disminuir las complicaciones inflamatorias pulmonares y posiblemente la mortalidad.

\section{Prevención}

En ausencia de una vacuna, las medidas de higiene y el distanciamiento social son las medidas de prevención másimportantes para contener esta epidemia. El uso adecuado de mascarillas, el lavado correcto y frecuente de manos, así como el uso de gel antiséptico son importantes. El distanciamiento social disminuye el contacto $y$, por lo tanto, la transmisión. Esta medida se debe aplicar en todos los niveles: individual, familiar o a nivel comunitario con la limitación en la asistencia laboral, en transporte o mediante el cierre de lugares de consumo y áreas públicas. La ciudad de Wuhan, donde se inició la epidemia, fue aislada por el gobierno de tal forma que hubiera distanciamiento entre la ciudad en donde ocurrían los casos y las demás ciudades de China. Este mismo método se ha replicado en algunas ciudades de Europa y Estados Unidos.

Finalmente, es de particular importancia la protección del personal de salud en los hospitales y centros de salud. En ellos se deben extremar las medidas de higiene y el correcto uso de equipos de protección personal en zonas de valoración médica externa, pero sobre todo en las áreas de hospitalización o de terapia intensiva. Es estas áreas se debe tener particular atención en las medidas de prevención por contacto: uso de guantes y bata, y en el caso de este particular virus, la protección ocular con caretas o goggles y ante gotas con el uso de máscara o careta quirúrgica. Cuando se realizan procedimientos quegeneran aerosoles (uso de equipos de apoyo ventilaiorio) existe la posibilidad de transmisión por vía aérea, por loque sedeben utilizar respiradores N95 o equivalentes. La frecuente higiene o lavado de manos es de fundamental importancia, no sólo en el área clínica, también en el domicilio, así como la limpieza de superficies, principalmente las de toque frecuente.

\section{Cuestionamientos pendientes}

Aún quedan muchas preguntas sin responder sobre esta nueva enfermedad. ${ }^{21}$ Persisten dudas sobre el comportamiento epidemiológico en diferentes entornos, así como sus formas de contagio y medidas eficaces de prevención. La información sobre la detección de anticuerpos en grandes poblaciones ayudará a establecer la prevalencia de la enfermedad, pero ayudará también a establecer el grado y la permanencia de la inmunidad ante la posibilidad de reinfecciones. Asimismo, queda por aclarar cuál es la realidad sobre la interacción con diversos 
medicamentos antihipertensivos, antiinflamatorios e hipoglicemiantes. Se evaluará la respuesta a nuevos tratamientos antivirales o inmunomoduladores y la eficacia de las vacunas. Aún no tenemos claro cuál será el momento adecuado de reducir el distanciamiento social o reiniciar las actividades laborales y comerciales. En suma, se deberán determinar las acciones internacionales para evitar, en medida de lo posible, un brote infeccioso similar en el futuro.

\section{COVID-19 EN MÉXICO}

\section{Origen, evolución y estado actual}

El primer caso detectado en nuestro país ocurrió el 27 de febrero de 2020 en el Instituto Nacional de Enfermedades Respiratorias, en la Ciudad de México, en un paciente con antecedente de haber viajado a Italia; el primer fallecimiento ocurrió el día 18 de marzo. El 24 de marzo, con 475 casos confirmados, se decretó la Fase 2 de «contingencia sanitaria», con medidas más estrictas de distanciamiento social, confinamiento y restricción laboral. La propagación de la enfermedad ha abarcado hasta ahora a todos los estados de la República, con el mayor índice de casos en Ciudad de México, Baja California y Sinaloa. De acuerdo con los datos de la Secretaría de Salud, al 14 de mayo de 2020, se cuenta con 40,186 casos confirmados, 24,856 casos sospechosos y 9,378 casos activos. Se han reportado 8,544 casos en trabajadores de la salud con infección confirmada (21\% del total), con mayor afección de médicos (47\%) y enfermeras (35\%) y 111 fallecimientos en este sector. México es uno de los países con menor número de pruebas diagnósticas aplicadas a la población en el mundo, y se estima que, de acuerdo a varios modelos epidemiológicos, la cifra real de pacientes infectados asciende a varios cientos de miles en el país y seguramente la cifra de muertes por COVID-19 es mayor. Se han reportado hasta ahora 4,220 fallecimientos por esta causa, lo que aporta una letalidad general de 10.5\% (Figura 2). ${ }^{22}$ Nos encontramos aún en una fase de ascenso en la curva de la epidemia, en cuanto a la incidencia en el número de casos se espera llegue a su punto más alto a mediados o finales de mayo, con un descenso esperado en julio de $2020 .^{22}$

\section{Factores de riesgo, comorbilidades y mortalidad}

El promedio de edad de los pacientes en México es de tan sólo 45 años con predominancia (58\%) del género masculino. De los casos activos confirmados $39.8 \%$ ha tenido que ser hospitalizado y $5 \%$ son reportados en estado crítico. La tendencia de hospitalización y la mortalidadaumentan con la edad. La letalidad de los casos confirmados en el mes de mayo de 2020 es de $6.6 \%$ en menores de 60 años y hasta de $24 \%$ en mayores de 60 años de edad. De los pacientes que fallecieron, el promedio de edad fue de 58
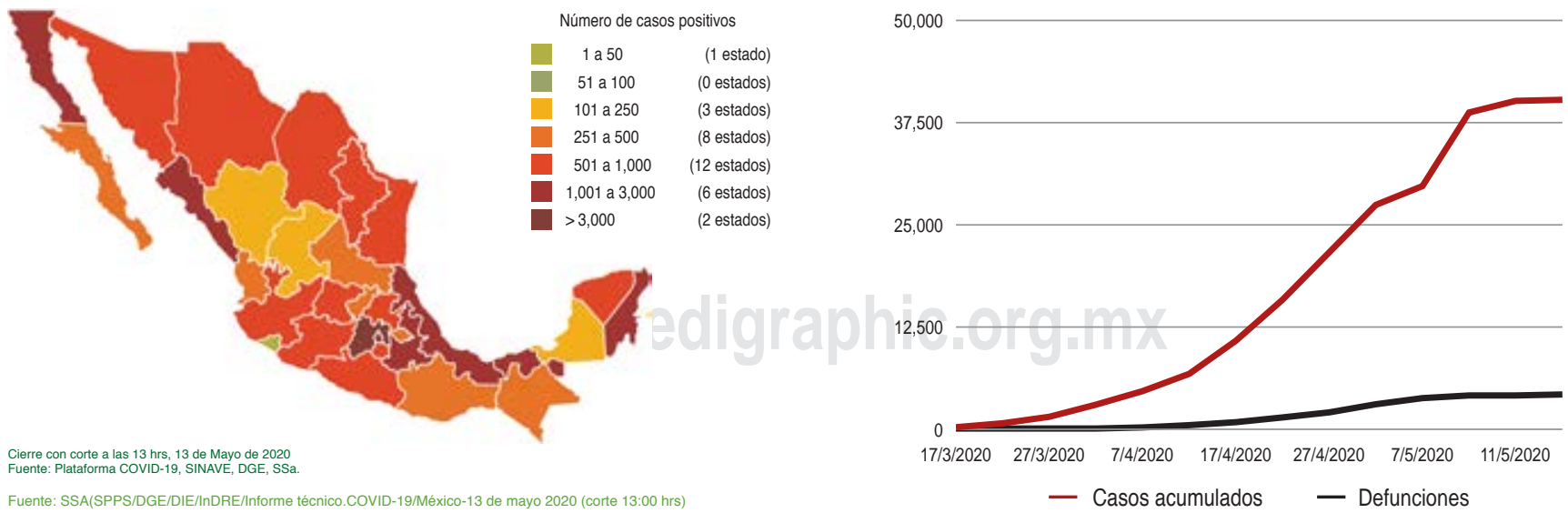

Figura 2. Mapa de número de casos confirmados por entidad federativa y gráfica de la evolución de casos confirmados y fallecimientos por COVID-19 en México (actualizado al 14 de mayo de 2020).22 
Tabla 2: Número, características y estado clínico de los pacientes con diagnóstico de COVID-19 en México (al 14 de mayo de 2020). ${ }^{22}$

$\begin{array}{cc}\text { Total de casos confirmados } & 40,186 \\ \text { Edad promedio (años) } & 46.0 \\ \text { Género masculino (\%) } & 58.2 \\ \text { Pacientes hospitalizados (\%) } & 39.8 \\ \text { Estado crítico (\%) } & 5.0 \\ \text { Personal de la salud infectado (n) } & 8,544 \\ \text { Médicos (\%) } & 47.0 \\ \text { Enfermeras (\%) } & 35.0 \\ \text { Otros (\%) } & 18.0 \\ \text { Total de pacientes confirmados fallecidos } & 4,220 \\ \text { Edad promedio (años) } & 58.0 \\ \text { Género masculino (\%) } & 68.2 \\ \text { Hipertensión (\%) } & 42.5 \\ \text { Diabetes (\%) } & 39.0 \\ \text { Enfermedad cardiovascular (\%) } & 6.3\end{array}$

años, $68.2 \%$ fueron hombres, $42.5 \%$ tenían hipertensión arterial, 39\% diabetes mellitus, 28.6\% obesidad, $9.6 \%$ tabaquismo, $10.5 \%$ enfermedad pulmonar obstructiva crónica, $7.2 \%$ insuficiencia renal crónica y $6.3 \%$ historia de enfermedad cardiovascular (Tabla 2). ${ }^{22}$

\section{Implicaciones en salud y socioeconómicas del COVID-19 en México}

El impacto de esta enfermedad será enorme en diferentes ámbitos y lo será aún más para un país como el nuestro. A corto plazo, si el ascenso de la curva de frecuencia de casos no se contiene, el sistema de salud corre el riesgo de verse rebasado, con altos costos presupuestales e importantes carencias en infraestructura, equipos y medicamentos. A mediano plazo el impacto social y psicosocial también serán notorios. Sin embargo, lo más preocupante es el ámbito económico a mediano y largo plazo. Se pronostica una inflación al alza, y las varias calificadoras internacionales han estimado no sólo un limitado crecimiento del Producto Interno Bruto (PIB), sino un decrecimiento de la economía. De acuerdo con la Organización para la Cooperación y Desarrollo Económico (OCDE), México será de las naciones más afectadas por esta crisis, con grandes implicaciones socioeconómicas y, por supuesto, en la salud. ${ }^{23}$
El mundo está viviendo una crisis sin precedente en este siglo. Saldremos adelante de esta fase, pero de ella aprenderemos mucho. Vendrá el dolor de la enfermedad y de la pérdida. Valoraremos el compromiso en la atención médica a costa de un riesgo personal. Aparecerán nuevos medicamentos y vacunas. No obstante, aprenderemos a cuidarnos mejor y a cuidar de los demás. Revaloraremos la higiene y la distancia. Aprenderemos nuevas tecnologías de telecomunicación y apreciaremos la paz del confinamiento y el valor del tiempo.

Como nos recordó acertadamente John $\mathrm{F}$ Kennedy: «La palabra 'crisis' escrita en chino está compuesta de dos caracteres. El primero: Wei (危) representa riesgo, y el segundo: Chi (机) oportunidad».

\section{REFERENCIAS}

1. Stern AM, Markel H. International efforts to control infectious diseases. J Am Med Assoc. 2004; 292: 1474-1479.

2. Frank TD, Carter A, Jahagirdar D, Biehl MH, DouwesSchultz D, Larson SL et al. Global, regional and national incidence, prevalence and mortality of HIV 1980-2017 and forecast to 2030 for 195 countries and territories: A systematic analysis for the burden of disease, injuries and risk factors study 2017. J Am Med Assoc. 2019; 6: e831-e859.

3. Del Río C, Mehta AK, Lyon GM 3rd, Guarner J. Ebola hemorrhagic fever in 2014: a tale of an evolv-ing epidemic. Ann Intern Med. 2014; 161: 746-748.

4. Lowe R, Barcellos C, Brasil P, Cruz OG, Álvez Honorio $\mathrm{N}$, Kuper $\mathrm{H}$ et al. The Zika virus epidemic in Brazil: From discovery to future implications. Int J Environ Res Public Health. 2018; 15: 1-18.

5. World Health Organization. The fifth meeting of the Emergency Committee under the International Health Regulations (2005) regarding microcephaly other neurological disorders and Zika virus. November 18, 2016. http://www.who.int

6. Huang C, Wang Y, Li X, Ren L, Zhao J, Hu Y et al. Clinical features of patients infected with 2019 novel coronavirus in Wuhan, China. Lancet. 2020; 395: 497-506.

7. Wu F, Zhao S, Yu B, Chen YM, Wang W, Song ZC et al. A new coronavirus associated with hu-man respiratory disease in China. Nature. 2020; 579: 265-269.

8. Del Río C, Melani PN. COVID-19 New insights of a rapidly changing epidemic. J Am Med Assoc. 2020; 323: 1339-1340.

9. Sanche S, Lin YT, Xu C, Romero-Severson E, Hengartner N, Ke R. High contagiousness and rapid spread of severe acute respiratory syndrome coronavirus 2 [published online ahead of print, 2020 Apr 7]. 
Emerg Infect Dis. 2020; 26 (7): doi: 10.3201/ eid2607.200282.

10. Zou F, Yu T, Du R et al. Clinical course and risk factors for mortality of adult inpatients with adult COVID-19 in Wuhan, China: a retrospective cohort study. J Am Med Assoc. 2020; 395: 1054-1062.

11. Guan WJ, Ni ZY, Hu Y et al. Clinical characteristics of coronavirus disease 2019 in China. N Engl J Med. 2020; 382 (18): 1708-1720.

12. Ministerio de Salud de España. Enfermedad por coronavirus (COVID-19). Actualización 26 de marzo de 2020 (Versión 2). [Accesado 10 de abril de 2020] Disponible en: https://mscbs.es/ccays/alertasActual/ nCov-China

13. European Centre of Disease Control and Prevention. Coronavirus disease 2019 (COVID-19) in the EU/EEA and the UK, 8th Update. [Accesado 10 de abril de 2020] Available in: https://ecdc. europa.eu.

14. Wu Z, McGoogan JM. Characteristics of and important lessons from the coronavirus disease 2019 (COVID-19) outbreak in China: summary of a report of 72314 cases from the Chinese Center for Disease Control and Prevention [published online ahead of print, 2020 Feb 24]. JAMA. 2020. doi: 10.1001/ jama.2020.2648.

15. World Health Organization. Coronavirus disease (COVID-19) pandemic. [Accesado 14 de abril de 2020] Available in: https://www.who.int/emergencies/diseases/novel-coronavirus-2019

16. Babiker A, Myers CW, Hill CE, Guarner J. SARSCoV-2 testing. Am J Clin Pathol. 2020; 153 (6): 706-708. doi: 10.1093/ajcp/aqaa052.

17. World Health Organization. Laboratory testing for coronavirus disease 2019 (COVID-19) in suspected human cases. Interim guidance 2 March 2020. [Accesado el 12 de abril de 2020] Available in: https:// apps.who.int/iris/handle/10665/331329

18. Wölfel R, Corman VM, Guggemos W, Seilmaier M, Zange S, Müller MA et al. Virological assessment of hospitalized patients with COVID-2019. Nature.
2020; 581 (7809): 465-469. doi: 10.1038/s41586020-2196-x.

19. Roden DM, Harrington RA, Poppas A, Russo AM. Considerations for drug interactions on QTc interval in exploratory COVID-19 treatment. J Am Coll Cardiol. 2020; 75 (20): 2623-2624. doi: 10.1016/j. jacc.2020.04.016.

20. Cao B, Wang Y, Wen D, Liu W, Wang J, Fan G et al. A trial of lipinavir-ritonavir in adults hospitalized with severe COVID-19. N Engl J Med. 2020. doi: 10.1056/NEJMoa2001282.

21. Omer SB, Malani P, Del Rio C. The COVID-19 pandemic in the US: a clinical update [published online ahead of print, 2020 Apr 6]. JAMA. 2020. doi: 10.1001/jama.2020.5788.

22. Secretaría de Salud de México. Dirección General de Epidemiología. Comunicado Técnico diario. [Accesado el 14 de abril del 2020] Disponible en: http://www.gob.mx/salud/documentos/coronaviruscovid-19

23. Laurence Boone. OECD Interim Outlook. Coronavirus: The world economy at risk. March 02, 2020. Available in: http://www.oecd.org/economy/outlook

\section{Correspondencia:}

Xavier Escudero (FACC, FSCAI, PMESC)

E-mail: xescuderodr@gmail.com

\section{RESPONSABILIDADES ÉTICAS}

Protección de personas y animales. LoS autores declaran que para esta investigación no se han realizado experimentos en seres humanos ni en animales.

Confidencialidad de los datos. Los autores declaran que han seguido los protocolos de su centro de trabajo sobre la publicación de datos de pacientes. 